Revue d'histoire de l'Amérique française

REVUE D.HISTOIRE DE L'AMÉRIQUE FRANÇAISE

\title{
L'état et le commerce de la morue de Terre-Neuve en France au XVIII ${ }^{\mathrm{e}}$ siècle
}

\section{Jean-François Brière}

Volume 36, numéro 3, décembre 1982

URI : https://id.erudit.org/iderudit/304066ar

DOI : https://doi.org/10.7202/304066ar

Aller au sommaire du numéro

Éditeur(s)

Institut d'histoire de l'Amérique française

ISSN

0035-2357 (imprimé)

1492-1383 (numérique)

Découvrir la revue

Citer cet article

Brière, J.-F. (1982). L'état et le commerce de la morue de Terre-Neuve en France au XVIII ${ }^{\mathrm{e}}$ siècle. Revue d'histoire de l'Amérique française, 36(3), 323-338.

https://doi.org/10.7202/304066ar d'utilisation que vous pouvez consulter en ligne.

https://apropos.erudit.org/fr/usagers/politique-dutilisation/ 


\title{
L'ÉTAT ET LE COMMERCE DE LA MORUE DE TERRE-NEUVE EN FRANCE AU XVIIIT SIËCLE
}

\author{
JEAN-FRANÇOIS BRIÈRE \\ professeur-assistant \\ Département de français \\ State University of New York at Albany
}

L'intérêt des historiens de l'Amérique française s'est, on le sait, orienté dans deux directions principales: l'histoire du commerce de la France avec ses colonies antillaises et celle de la colonisation française sur le continent américain. La pêche terreneuvière métropolitaine, qui n'a jamais mis en jeu un capital financier comparable à celui du commerce des Iles et ne fut à l'origine d'aucune colonisation d'envergure, a longtemps été maintenue dans un relatif oubli par les historiens de langue française ${ }^{1}$. Certes, les tribulations du conflit franco-britannique à propos du French Shore sont bien connues ${ }^{2}$. Mais on ne saurait en dire autant de l'armature économique et sociale de la pêche terreneuvière. Quelques travaux récents ou en cours commencent heureusement à jeter une lumière nouvelle sur ces questions ${ }^{3}$.

1 La seule étude récente de quelque ampleur publiée en langue française sur ce sujet ne vient pas d'un historien professionnel: Charles de la Morandière, Histoire de la pêche française de la morue dans l'Amérique septentrionale (Paris, Maisonneuve \& Larose 1962), 3 vol.

2 Voir par exemple: Anonyme, Notice historique et documentaire sur la question du French Shore à Terre-Neuve, 1713-1899 (Saint-Malo, 1899) 38 p. - R. de Caix, TerreNeuve, Saint-Pierre et le French Shore; la question des pêcheries et le traité du 8 avril 1904 (Paris, Société française d'imprimerie et de librairie, 1904) 100 p. - E.T. Daubigny, Choiseul et la France d'outre-mer après le traité de Paris: étude sur la politique coloniale au $X V I I I e$ siecle, avec un appendice sur les origines de la question de Terre-Neuve (Paris, Hachette, 1892) 352 p. - E. Hervé, Le French Shore et l'arrangement du 8 avril 1904 (Rennes, Eugène Prost, 1905) 160 p. - C. de La Roncière, La question de Terre-Neuve. Les droits indiscutables de la France (Paris, De Soye \& Fils, 1904) 36 p.

En anglais: R.L. Dashwood, The French in Newfoundland (London, 1899) 24 p. - C. Isham, The Fishery Question; its Origin, History and Present Situation (New York \& London, Putnam's Sons, 1887) 89 p. - J. Jay, The Fisheries Dispute (New York, Dodd, Mead and Co, 1887) 52 p. - P. Neary, "The French and American Shore Questions as Factors in Newfoundland History", Newfoundland in the 19th and 20th Centuries, J. Hiller and P. Neary ed. (Toronto, University of Toronto Press, 1980): 95-119. - F.F. Thompson, The French Shore Problem in Newfoundland (Toronto, U. of Toronto Press, 1961).

3 Voir J.F. Bosher, «A Fishing Company of Louisbourg, Les Sables d'Olonne and Paris: la Société du Baron d'Huart, 1750-1775», French Historical Studies, IX, 2 (Fall 1975): 263-277. - J.F. Briere, L'Armement français pour la pêche à Terre-Neuve au $X$ VIIIe siècle, thèse de Ph.D. (York University, 1980), dactyl., 442 p. - J.F. Briere, «Le trafic terre-neuvier malouin dans la première moitié du XVIIIe siècle (1713-1775)», Histoire sociale/Social History, XI (nov. 1978): 356-374. - C. Carrière, Les négociants marseillais au XVIIIe siecle (Marseille, 1974) 2 vol., 1110 p. - R. Richard, "Comptes et profits de

RHAF, vol. 36, no 3, décembre 1982 
L'évolution du système fiscal appliqué pendant le XVIIIe siècle aux armements terre-neuviers français et à leurs cargaisons de morue est intéressante non seulement du point de vue strictement commercial, mais aussi sur le plan des rapports entre le politique et l'économique dans la mesure où elle reflète une attitude nouvelle des dirigeants de l'État à l'égard de la pêche. Deux forces distinctes additionnèrent leurs efforts pour produire cette mutation: la vulgarisation des idées libérales en matière économique - et surtout fiscale - d'une part, la crainte de voir disparaitre la pêche terreneuvière française de l'autre. En effet, le gouvernement français qui, en 1763, réussit à conserver à la France la mer de Terre-Neuve, part jugée essentielle de l'Amérique française, redouta constamment par la suite d'en voir son pays éliminé sous la pression de la concurrence britannique. La conjoncture politique et commerciale nord-américaine allait, par ce détour, peser lourdement sur l'évolution de la fiscalité de la pêche morutière en France.

Lorsque la France, au XVIIIe siècle, entamait une guerre sur mer, sa marine royale devait être en mesure de recruter très rapidement un personnel considérable dont la formation technique, en raison de sa longueur, ne pouvait pas s'improviser au dernier moment. Elle prenait donc ses recrues parmi les matelots déjà formés à la navigation, lesquels étaient astreints au service obligatoire suivant le système des classes mis en place sous Colbert. La marine civile constituait ainsi le réservoir de main-d'oeuvre où la marine militaire puisait librement en fonction de ses moyens et de ses besoins. La pêche terre-neuvière occupait une place importante dans ce réservoir humain en raison du nombre considérable d'hommes qu'elle employait - 10 à 15000 simultanément - et de la qualité de leur entraînement. Ce système aboutissait à lier certains éléments de la puissance navale de la France à la fortune commerciale d'un certain nombre d'armateurs; car si la pêche terreneuvière déclinait ou s'effondrait en tant qu'activité rentable, le réservoir d'hommes très aguerris dans lequel la marine pouvait puiser risquait de disparaître. Le pouvoir royal ne pouvait donc rester totalement indifférent au sort des négociants dont les initiatives personnelles soutenaient une activité hautement utile à l'État. On constate ainsi, pendant tout le XVIIIe siècle, un certain parallélisme entre l'attention portée par les gouvernants aux problèmes de la puissance navale et l'intérêt qu'ils marquaient à ce que le commerce morutier s'exercât dans des conditions de rentabilité

navires terre-neuviers du Havre au XVIIe siècle (aspects économiques et sociaux)», Revue d'histoire économique et sociale, 54, 1976 (4): 476-524. - L. Turgeon, «Pour une histoire de la pêche: le marché de la morue à Marseille au XVIIIe siècle», Histoire sociale/Social History, XIV (nov. 1981): 295-322. 
satisfaisantes. Les armateurs terre-neuviers, de leur côté, étaient parfaitement conscients du rôle qu'ils occupaient dans le système et des moyens de pression que cette position leur offrait; ils n'hésitaient jamais à s'en servir pour forcer la main au pouvoir royal.

Ces données propres au commerce terre-neuvier allaient se combiner avec le libéralisme ambiant du siècle pour provoquer un renversement complet des rapports entre l'État et ce commerce. Non seulement le poids de la fiscalité pesant sur le commerce morutier allait être progressivement allégé, mais on verrait même apparaître une véritable "fiscalité inversée» destinée à soutenir l'activité terre-neuvière à l'aide des deniers publics.

\section{Les droits sur la morue}

Au début du XVIIIe siècle, les bureaux du secrétariat d'État à la Marine ne possédaient que des connaissances très vagues et morcelées sur la pêche terre-neuvière. Les statistiques envoyées par les commissaires des classes restaient souvent imprécises et incomplètes. On n'essayait jamais de les regrouper afin d'obtenir une vue d'ensemble de l'armement morutier. L'attitude du pouvoir était fondamentalement «fiscaliste», c'est-à-dire que tout en étant conscient de l'importance de Terre-Neuve pour la marine royale, il ne s'intéressait au déroulement de la pêche et à ses résultats que dans la mesure où la pêche était l'objet d'un prélèvement fiscal de sa part: au moment de l'armement, quand une retenue était effectuée sur les avances versées aux équipages pour le financement du service des Invalides de la Marine et lorsque la morue rapportée devait payer des droits. Une telle conception explique pourquoi les statistiques morutières de l'époque portaient toujours sur le déchargement des cargaisons. Les bureaux de Versailles connaissaient avec plus ou moins d'exactitude les quantités de poisson livrées dans chaque port, mais ignoraient - et ne faisaient aucun effort - pour connaître les prises de chacun de ces ports.

Les droits qui frappaient le commerce de la morue étaient fort nombreux. Les droits de gabelle payés à l'achat du sel dans les ports situés en pays non-exemptés et les droits de sortie des traites sur les marchandises d'avitaillement étaient levés avant le départ des navires. Au retour, la cargaison de morue se voyait soumise à une série de droits royaux ou privés d'autant plus nombreux que la destination du poisson était éloignée du port de débarquement. Mais nombreux ne signifie pas énorme; en effet, la fiscalité à laquelle était soumis le commerce de la morue en France ne semble pas avoir été aussi lourde que les négociants cherchaient toujours à le laisser croire. Un mémoire nantais de 1738 nous indique qu'un 
millier de morue verte "grande et marchande» (1 240 morues) expédié de Nantes à Paris via Orléans devait acquitter les droits suivants: ${ }^{4}$

1) à Nantes:

- Prévôté

- Quatre sols pour livre du droit précédent

- Octrois de Nantes

- Traite domaniale

- Certificat et formules

$11.1 \mathrm{~s}$.

33 1. 6 s. 8 d. $6 \mathrm{~s}$.

2) à Ingrande (entrée dans le ressort de la Ferme Générale):

- Entrée de France

- Abord

- Consommation

- Quatre sols pour 1. des trois droits précédents

- Concédés

- Officiers et signatures

- Parisis

- Acquit, certificat et formule

- Simple Cloison d'Angers

- Double et triple Cloison d'Angers

- Boete

- Trépas de Loire

$91.6 \mathrm{~s}$. 33 1. 12 s. 6 d. 40 l. 6 s. 8 d.

3) au Pont de Cé:

$161.3 \mathrm{~s}$. 101.

$11.15 \mathrm{~s}$.

11 s. $8 \mathrm{~d}$. 14 s. $6 \mathrm{~d}$.

$21.3 \mathrm{~s}$.

$41.19 \mathrm{~s}$.

11.6 s. 6 d.

21.4 s. 4 d.

- Officiers et signatures

- Prévôté d'Angers (à l'évêque d'Angers)

4) à Saumur:

- Officiers et signatures

- Prévôté de Saumur (à l'abbé de Fontevrault)

$11.18 \mathrm{~s}$.

11.1 s. 8 d.

$11.18 \mathrm{~s}$.

$18 \mathrm{~s}$.

5) à Orléans:

- Péage d'Orléans

$11.5 \mathrm{~s}$.

Les auteurs du mémoire précisent que le prix de revient de ce millier de morue, droits exclus, s'établissait à environ $1220 \mathrm{l}$. (1000 l. pour l'achat, $20 \mathrm{l}$. de commissions diverses à l'achat, 200 1. pour le transport de Nantes à Orléans). Le total des droits perçus se montant à 2021 . (201 $1.19 \mathrm{~s} .10 \mathrm{~d}$. exactement), il en ressort qu'entre l'achat à Nantes et l'arrivée à Orléans, le prix de revient du millier de morue s'était accru de 42\% (de $1000 \mathrm{l}$. à

\footnotetext{
4 Archives départementales Loire-Atlantique, C744, 100 bis, «Etat des droits perçus le long de la Loire et à Paris sur un millier, compte marchand, de morue verte grande et marchande», 18 octobre 1738.
} 
1422 1.) dont $20 \%$ dus à la seule fiscalité. Si la cargaison était ensuite voiturée d'Orléans à Paris, il fallait ajouter:

- Frais de transbordement et commissions à Orléans

- Voiture d'Orléans à Paris

- Frais de décharge et de vente à Paris

et les droits suivants à Paris:

- Droit de Bureau sur la vente

- $15 \%$ du droit de Bureau à l'Hôpital général de Paris

301.

1701.

$71.10 \mathrm{~s}$.

330 1. 8 s. 8 d.

161.12 s. 4 d.

On retranchait alors le montant du droit de consommation (et sols pour livre) - 48 1. $8 \mathrm{~s}$. - dont Paris était exempté. Le millier de morue verte acheté $1000 \mathrm{l}$. à Nantes revenait ainsi à $19281.3 \mathrm{~s}$. lors de son arrivée à Paris: presque le double. Les auteurs, qui réclamaient des exemptions, insistaient lourdement sur ce monstrueux gonflement; mais ils nous indiquent aussi, presque à la sauvette, le prix de vente moyen de ce millier de morue: 2600 l. Il en résulte que le prix d'achat à Nantes représentait $38,4 \%$ du prix de vente à Paris, les frais de transport (427 1.) $16,4 \%$, les droits $(5001) 19,.2 \%$, et le profit du marchand $(6721$. $25,8 \%$. Le poids fiscal, lourd certes, ne semble pas avoir été exorbitant. Une brusque suppression de tous les droits sur la morue aurait à peine fait baisser d'un cinquième le prix de la morue à Paris. On ne peut donc qu'être très sceptique lorsque les consuls de Nantes affirment que les quantités de morue vendues «quintupleroient si les droits étoient réduits au cinquième de leur montant $\rangle^{5}$. Les négociants, comme les économistes de l'époque, surestimaient presque toujours les effets à attendre d'une suppression des droits sur l'expansion du marché. Ils sous-estimaient l'importance des autres facteurs en cause, notamment les limitations techniques et le coût du transport, dont on ne pouvait encore concevoir de modifications profondes. Seuls les droits paraissaient élastiques et compressibles à volonté. Ici comme ailleurs, le problème fondamental de la fiscalité d'Ancien Régime semble avoir été celui d'un système plus arbitraire et irrationnel qu'écrasant par son poids.

Le montant des droits frappant le commerce de la morue était déterminé non pas à partir de la valeur marchande réelle du produit, mais - suivant l'usage de l'époque - à partir des quantités transportées (cents ou milliers de morues, etc...). Il en découle que la part de la fiscalité dans le prix de vente de la morue aux consommateurs n'était pas stable, mais variait en fonction inverse du prix

5 Ibid. 
du poisson dans les ports de déchargement. Plus le prix de la morue était bas dans ces ports, plus la part des droits et des frais de transport dans le prix de vente allait être élevée. Le poids relatif de la fiscalité variait aussi suivant la dimension et la qualité du poisson puisque celui-ci était imposé à la pièce: les mêmes droits, modérés sur le grand poisson de bonne qualité, devenaient prohibitifs lorsqu'on les appliquait au poisson de faible dimension ou en mauvais état. Les armateurs des Sables d'Olonne notaient ainsi, en 1783 , qu'en raison de la fiscalité, seule la grande morue pouvait être expédiée dans le «pays haut»; or, cette qualité, ajoutaient-ils, ne représentait jamais plus du quart d'une cargaison. Conséquence: le moyen et le petit poisson pourrissaient sur les quais des ports de déchargement, faute de pouvoir être entièrement absorbés par le marché local. Rien d'étonnant, dans ces conditions, à ce que la morue verte ait été une denrée de consommation populaire dans la région nantaise et un plat pour riches à Paris. Fiscalité et coût du transport s'alliaient ainsi pour freiner la propagation des bas prix vers l'intérieur du pays. Un tel mécanisme contribuait à aggraver les phénomènes d'engorgement et d'effondrement des cours qui se manifestaient dans les ports de déchargement au lendemain des pêches très fructueuses.

\section{Les exemptions (1664-1730)}

Les armateurs terre-neuviers n'avaient pas attendu le XVIIIe siècle pour obtenir des exemptions fiscales. Un premier type d'exemption se rapporte aux droits d'entrée sur le poisson. Le tarif de 1664 confirma aux Havrais et aux Dieppois une réduction des droits d'entrée dont ils jouissaient déjà depuis fort longtemps ${ }^{6}$. L'année suivante, Honfleur obtenait aussi une telle réduction ${ }^{7}$. D'autres ports normands reçurent de semblables privilèges. Au début du XVIIIe siècle, les droits d'entrée payables sur les morues déchargées au Havre variaient ainsi en fonction du port d'attache du bâtiment qui les livrait ${ }^{8}$ :

\section{Port d'attache}

Le Havre, Dieppe, Fécamp,

St Valéry-en-Caux

Honfleur, Granville

St-Malo et autres ports de

Bretagne (droits pleins) droit d'entrée
cent de $m$. verte

$4 \mathrm{~s}$.

$12 \mathrm{~s}$.

3 livres droit d'entrée

cent de $m$. sèche

$2 \mathrm{~s}$.

$6 \mathrm{~s}$.

2 livres

AD Loire-Atlantique, C744, 68, arrêt du Conseil du 31 décembre 1664.

AD Loire-Atlantique, C744, 65, arrêt du Conseil du 22 janvier 1665.

8 AD Loire-Atlantique, C744, 67, «Etat des droits payés au Havre pour les morues vertes, sèches et les huiles qui y sont déchargées» (s.d., début XVIIIe siècle). 
Ces différences de traitement sont énormes. Il est difficile de savoir si la défaveur relative dont souffraient les Bretons était une cause ou une conséquence de leur spécialisation dans l'armement à la morue sèche. Tant que le commerce de celle-ci leur suffit, ils n'eurent rien à redire à cet inconvénient fiscal; les Normands ne les concurrençaient pas sur leurs marchés méridionaux. Ajoutons à cela que le montant des droits ne variait pas seulement en fonction des ports d'armement, mais aussi selon l'endroit où la morue avait été pêchée: à partir de 1713 , en effet - et jusqu'en 1763 - les morues sèches provenant de l'île Royale purent être introduites en France en exemption de tous droits d'entrée 9 .

Dès 1665, les armateurs des Sables d'Olonne avaient obtenu l'exemption de tous droits de sortie des traites sur les marchandises d'avitaillement consommées sur leurs terre-neuviers ${ }^{10}$. Ils furent les seuls à en jouir, semble-t-il, jusqu'aux lettres patentes d'avril 1717 qui octroyèrent une semblable exemption à tous les ports autorisés à commercer avec les colonies d'Amérique pour leurs armements avec ces colonies ${ }^{11}$. Deux restrictions importantes limitaient la portée de ces lettres: d'une part, ni Granville ni SaintJean-de-Luz, importants ports terre-neuviers, n'étaient compris parmi les ports autorisés à commercer avec les colonies; d'autre part, les lettres ne s'appliquant qu'aux expéditions pour les colonies françaises, ni la pêche errante sur les bancs ni la pêche sédentaire sur les côtes de Terre-Neuve ne pouvaient bénéficier du privilège accordé. Cette inégalité de traitement a certainement pesé sur la réorientation de la pêche malouine vers le continent canadien après 1717.

Un troisième type de réduction de droits s'appliquait aux sels, denrée hautement sensible sur le plan fiscal, et pour cette raison soumise à un régime spécial d'exemptions. Le titre 15 de l'ordonnance des Gabelles de 1680 autorisait les terre-neuviers des villes maritimes de Normandie et de Picardie à aller prendre leur sel aux marais de Brouage en exemption de tout impôt, à condition - précaution indispensable - qu'ils se rendent directement à TerreNeuve une fois leur sel embarqué ${ }^{12}$. L'administration royale veillait attentivement à ce que ce sel ne soit pas revendu en France lors de son retour avec les morues. Les sels de retour, considérés comme inutilisables pour la pêche, devaient être immergés, mais il fallait en garder la quantité minimale nécessaire à la conservation

9 AD Loire-Atlantique, C744, 59, arrêt du Conseil du 9 septembre 1713.

10 AD Loire-Atlantique, C744, 5, arrêt du Conseil du 12 avril 1723. L'arrêt d'exemption est du 17 juin 1665 .

11 AD Loire-Atlantique, C744, 9, lettres patentes avril 1717.

12 La Morandière, Histoire de la pêche française de la morue dans l'A mérique septentrionale (Paris, 1962), 2: 973. 
du poisson durant son transport à travers le pays. Des frictions permanentes opposaient l'administration aux marchands de morue à ce sujet: en octobre 1713, Michel Lallemant de Betz et son collègue Le Couturier, contrôleurs des Fermes à Ingrande et à $\mathrm{La}$ Pointe, étaient sommés par le contrôleur général Desmarets de «veiller exactement à ce que les gardes secouent sufisament les morues qui passent sur la Loire affin qu'il n'y reste qu'autant de sel qu'il faut pour les conserver (...) sy je recois à l'avenir des plaintes sur le faux saunage qui se fait au moyen des sels que les voituriers chargés de morues répandent le long de la Rivière de Loire, je l'attriburay uniquement à leur négligence (...) ${ }^{13}$. Menace, comme beaucoup d'autres, sans effet: en février 1715, tous les gardes «prennent de l'argent des voituriers pour donner la main au faux saunage (...) ils débittent icy ce sel-là aux boulangers et artisans. M. Chenart devroit bien arrester ce desordre (...) ${ }^{14}$. Privées d'une quantité suffisante de sel, les morues arrivaient souvent en mauvais état à destination. Les voituriers, coupables ou non, ne manquaient pas d'en accuser les gardes. D'où les plaintes des négociants: "Il semble par l'acharnement de ces supôts des fermes qu'ils ayent déclaré une guerre ouverte à cette malheureuse marchandise. ${ }^{15}$

À ces privilèges directs, il convient d'ajouter un avantage indirect dont bénéficia, pendant tout le XVIIIe siècle, la pêche française: la création, fin 1687, d'un droit d'entrée sur la morue étrangère de 21 . par cent de morue sèche (l'équivalent des droits perçus sur la morue française) et de 81 . sur chaque cent de morue verte (près de trois fois les droits perçus sur la morue française) ${ }^{16}$. Ces mesures visaient à bloquer les importations de morue anglohollandaise ${ }^{17}$. Le barrage fiscal semble avoir été placé trop bas pour le temps de guerre puisqu'en 1691 les droits étaient haussés: 41 . sur le cent de morue sèche, 121 . sur le cent de morue verte ${ }^{18}$. En 1701, les droits sur la morue anglaise furent doublés $(81$. le cent de morue sèche), puis, en 1703, la guerre entraîna son interdiction d'entrée à Marseille, port franc, où elle ne payait aucun droit ${ }^{19}$.

13 AD Loire-Atlantique, C744, 81, Desmarets à Du Ruaupallu, 12 octobre 1713.

14 AD Loire-Atlantique, C744, 85, anonyme à De Boiscorbon, 12 février 1715.

15 AD Loire-Atlantique, C744, 112, mémoire des négociants nantais au contrôleur général, 17 novembre 1772 .

16 Archives publiques du Canada, MG2 A1, liasse 24, 122, arrêt du Conseil du 20 décembre 1687. L'arrêt était applicable à partir du ler janvier 1688 .

17 Les provinces d' "étranger effectif" de l'est du royaume (Luxembourg, Trois Evêchés, Sarre, Alsace), dont tout l'approvisionnement en morue venait de Hollande par le Rhin étaient maintenues hors de ce barrage fiscal. Voir APC, MG2, A1, liasse 25, 21, arrêt du Conseil du 16 mars 1688 .

18 APC, MG2 A1, liasse 28, 110, arrêt du Conseil du 4 octobre 1691.

19 Archives de la Chambre de Commerce de Bayonne, 12, no 11, arrêt du Conseil du 6 septembre 1701 et Rambert, Histoire du commerce de Marseille (Paris, 1959), 487, arrêt du Conseil du 10 juillet 1703 . 
Au début du XVIIIe siècle, la pêche française bénéficiait donc de privilèges fiscaux importants. Certains d'entre eux provenaient de la situation originale de lá pêche terre-neuvière: lever des droits de gabelle sur les quantités énormes de sel nécessaires à TerreNeuve eût placé les coûts d'armement à un niveau difficilement soutenable; l'exemption de droits de sortie sur les marchandises d'avitaillement peut s'expliquer par le fait que la consommation de ces produits à bord de bâtiments ne faisant aucune escale à l'étranger était assimilée à une consommation intérieure. D'une manière générale, l'ensemble des privilèges accordés à la pêche ne formait pas un système logique et cohérent; c'était l'habit d'Arlequin habituel en la matière, composé de morceaux mal cousus ensemble: législation souvent trop vague, incomplète, parfois contradictoire, presque toujours mal appliquée. Les denrées d'avitaillement provenant de l'étranger étaient-elles aussi dispensées du paiement des droits de sortie? Nul ne le sut jusqu'en $1723^{20}$. Les cargaisons des terre-neuviers ennemis capturés devaient-elles payer les droits sur la morue étrangère? Les Fermiers généraux répondaient affirmativement, puisqu'elles étaient de pêche étrangère; les armateurs négativement, puisqu'elles appartenaient dorénavant à des Français ${ }^{21}$. Une curieuse source de malentendu, où la mauvaise foi ne devait pas être absente, opposa aussi l'administration des Fermes et les armateurs terre-neuviers à propos du sens exact du mot «royaume» dans la législation royale. Les fermiers donnaient à ce terme son sens fiscal de ressort des Cinq Grosses Fermes, excluant par là les provinces extérieures à ce territoire. Du côté des armateurs, au contraire, on estimait que le mot «royaume» était pris dans son sens politique de territoire assujetti, couvrant donc toutes les provinces françaises. On imagine aisément les difficultés que pouvait poser l'application de textes donnant lieu à de tels écarts d'interprétation. Dans certains cas, la législation royale n'était suivie d'aucun effet: le droit d'abord, supprimé sur toutes les cargaisons françaises de poisson par l'arrêt du 9 février 1671, était toujours perçu à Ingrande en $1738 . . .22$

L'évolution de ce vaste et complexe système de droits et de privilèges suivit, tout au long du XVIIIe siècle, deux orientations successives: tout d'abord il s'uniformisa avec la disparition progressive des inégalités de régime entre les différents ports; il se pro-

20 AD Loire-Atlantique, C744, 5, arrêt du Conseil du 12 avril 1723.

21 Voir à ce sujet l'arrêt du 7 août 1744 , qui autorise la vente du poisson salé provenant des prises de guerre moyennant le paiement d'un droit de 10\%; Rambert, Histoire du commerce de Marseille (Paris, 1959), 487.

22 AD Loire-Atlantique, C744, 100 bis, «Etat des droits...» (octobre 1738). L'auteur du document souligne l'illégalité de la perception. 
longea ensuite avec la création d'un système de primes versées par l'État aux armateurs pour le soutien de la pêche.

\section{La généralisation des exemptions (1730-1763)}

La dynamique de la pêche terre-neuvière au long du XVIIIe siècle fut caractérisée par le déclin d'un certain nombre de ports très actifs au siècle précédent (Dieppe, Le Havre, Honfleur, Nantes, Les Sables, La Rochelle, les ports basques) et l'essor de quelques autres auparavant moins bien placés (Saint-Malo, Granville). Ce double mouvement joua un rôle capital dans l'extension des privilèges fiscaux attribués à la pêche. En effet, le système hérité du XVIIe siècle tendait à favoriser les ports qui avaient été les plus développés à cette époque. Aussi, lorsque Granville, Saint-Malo, Saint-Brieuc, développèrent leurs activités terre-neuvières, notamment à partir des années 1730, leurs armateurs insistèrent pour être mis sur un pied d'égalité avec les ports privilégiés; ils prirent prétexte de l'importance de leur pêche pour obtenir réparation de l'«injustice» qui leur était faite. Quant aux négociants des ports anciennement privilégiés, ils jouèrent au contraire sur le déclin de leurs armements pour réclamer des privilèges supplémentaires. Les premiers utilisaient leur force, les seconds leur faiblesse afin d'obtenir les mêmes avantages: une réduction croissante du prélèvement fiscal opéré sur leur pêche.

L'offensive se déclencha dans les années 1730, aiguillonnée, semble-t-il, par les progrès rapides de la morue anglaise hors de ses marchés traditionnels - Portugal et Espagne atlantique - en direction de la Méditerranée occidentale. De 1732 à la guerre de Sept ans, les arrêts d'exemption se succédèrent à une cadence accélérée: en août 1732, les huiles de pêche française reçurent dispense de tous droits pour six ans, dispense régulièrement renouvelée par la suite ${ }^{23}$. En mars 1733, l'exemption de tous droits d'entrée sur la morue de l'île Royale fut prorogée pour dix ans ${ }^{24}$. Le 31 octobre de la même année, le bénéfice des lettres patentes de 1717 (exemption des droits de sortie sur l'avitaillement) fut étendu à l'ensemble

23 APC, MG2 A1, liasse 74, 47, arrêt du Conseil du 19 août 1732.

24 AD Loire-Atlantique, C744, 53, arrêt du Conseil du 17 mars 1733. L'arrêt donne la liste des droits dont la morue était exemptée: droits d'entrée des Cinq Grosses Fermes; sol pour livre à l'entrée des ports de Normandie et à Ingrande; prévôté; comptablie et courtage des ports de la sénéchaussée de Bordeaux; coutume de Bayonne; douane de Lyon; tiers surtaux et quarantiéme; table de mer; deux pour cent d'Arles. Afin de bénéficier de ces exemptions, les capitaines devaient faire déclaration de leur destination pour l'Ile Royale au Bureau des fermes. Le receveur leur donnait un extrait de cette déclaration qu'ils devaient faire viser par l'Amirauté de Louisbourg et présenter à leur retour au Bureau des Fermes pour preuve de leur destination. Ces formalités, bien entendu, n'empêchaient pas la fraude qui consistait à faire passer pour provenant de l'Ile Royale des cargaisons de poisson pêchées à Terre-Neuve ou même achetées aux Anglais. 
de la pêche terre-neuvière ${ }^{25}$. Aussitôt, les armateurs de Saint-Jeande-Luz demandèrent et obtinrent que leur ville soit ajoutée à la liste des ports jouissant du privilège: mesure indispensable, selon eux, puisqu'ils «ont fait des pertes considérables depuis quelques années dans leur pesche de la baleine et de la morue» ${ }^{26}$. Les Granvillais, dont la pêche sédentaire décollait, formulèrent alors la même demande, sous prétexte qu'ils commercaient avec le Canada depuis plus de deux cents ans ${ }^{27}$. Leur requête fut acceptée. En octobre 1738, les Nantais envoyèrent un long mémoire à Versailles pour obtenir une réduction des droits sur les morues envoyées vers Paris. Ce sont ces droits qui, d'après eux, étaient la cause du déclin de la pêche aux Sables et à Nantes: "Cens cinquante vaisseaux seroient occupés à cette pesche si les droits étoient proportionnés à la valeur de la marchandise, parce que tous les gens du royaume pourroient en manger (...)»

Pourquoi, suggèrent-ils, ne pas suivre l'exemple des Hollandais? Chez eux, le poisson entre sans payer de droit parce que la pêche y «est regardée comme la pierre fondamentale de l'etat»; il faut d'autant plus développer la consommation intérieure française et éliminer le poisson hollandais du marché français que depuis le traité d'Utrecht «les pêcheurs anglois se sont emparés de ce commerce et fournissent presque seuls de morue l'Espagne et l'Italie ${ }^{28}$. Les Nantais demandaient aussi que les droits perçus sur leurs morues portées au Havre soient réduits et alignés sur ceux auxquels sont assujetties les morues de pêche normande ${ }^{29}$. Sablais et Malouins se joignirent bientôt à eux pour formuler la même requête et «si cette demande leur étoit refusée, ils seroient forcez de renoncer à la pesche de la morue verte» ${ }^{30}$. Malgré l'opposition de la Chambre de Commerce de Rouen et des Fermiers généraux, Saint-Malo obtint gain de cause en juillet 1739, les Sables en avril $1740^{31}$. Les Normands, défavorisés sur le plan du sel, ne furent pas en reste: Granville et Honfleur obtinrent en janvier 1739 le droit de prendre leur sel en Bretagne, ce qui les mit sur un pied d'égalité avec Saint-Malo dans la course annuelle aux meilleurs emplacements de pêche ${ }^{32}$.

25 AD Loire-Atlantique, C744, 15, mémoire sur les productions de la Bretagne. L'arrêt est du 31 octobre 1733 .

26 APC, MG2 A1, liasse 72, 20, arrêt du Conseil du 20 juillet 1734.

27 APC, MG2 A1, liasse 76, 4, arrêt du Conseil du 27 janvier 1739.

28 AD Loire-Atlantique, C744, 100, mémoire des juges et consuls de Nantes, 18 octobre 1738 .

29 Ibid.

30 APC, MG2 A1, liasse 76, 25, arrêt du Conseil du 14 juillet 1739.

31 Ibid. et APC, MG2 A1, liasse 77, 11, arrêt du 5 avril 1740.

32 APC, MG2 A1, liasse 76, 2 et 3, arrêts des 13 et 27 janvier 1739. 
Toutes ces nouvelles mesures ne furent obtenues qu'au prix d'un siège en règle des bureaux de Versailles. Bouhier de l'Ecluse, député du Commerce des Sables, nous a laissé un compte rendu vivant et significatif de l'entrevue qu'il eut le 13 août 1739 avec l'intendant Fagon, chargé des Cinq Grosses Fermes au Contrôle général, à propos des réductions de droits sur la morue:

Je lui dis (...) voilà un mémoire instructif de ce grief et de beaucoup d'autres, Monseigneur, que j'ai l'honneur de vous prêter - Je n'en ai pas besoin, m'a-t-il répondu (...) Et que demandez-vous donc, m'a-t-il dit? - La suppression, Monseigneur, de plusieurs autres droits déjà supprimés par l'édit de 1664 et qui ont été inclus mal à propos dans le tarif. Il s'est mis à sourire un peu amèrement. Monseigneur, lui ai-je dit, notre commerce ne sauroit se soutenir si on ne supprime cette multiplicité de droits qui empeschent les bons marchands d'acheter notre morue par les sommes considerables qu'il leur faut debourser pour les acquitter, l'inquietude d'esprit que cela leur donne et la crainte de tomber en contravention; nous sommes ruinés et nous allons abandonner notre commerce si vous n'avez pas la bonté de nous rendre justice - Bon, a-t-il dit ironiquement, ils vont abandonner leur commerce (...) Eh bien, Monsieur, il faudra examiner tout cela et vous rendre justice - Je vous la demande prompte, Monseigneur, s'il vous plaît et je vous supplie très humblement de considérer que je suis ici aux frais d'une communauté pauvre - Eh! que faites-vous ici, Monsieur, m'a-t-il dit avec vivacité, allez-vous en, on vous rendra justice comme si vous étiez présent (...) Voilà la première bourrasque qu'il m'a fallu essuyer, nous nous aguerirons peu à peu (...) si je quitte la partie, je la perds. ${ }^{33}$

Cette vague d'exemptions fut complétée en mai 1742 par une réduction d'un tiers des droits d'entrée des Cinq Grosses Fermes sur les morues sèches: $3 \mathrm{~s}$. le «cent pesant» au lieu des $3 \mathrm{l}$. par millier marchand du tarif de $1664^{34}$.

\section{Les primes (1763-1792)}

La seconde grande vague d'exemptions déferla au lendemain de la guerre de Sept ans. Elle fut provoquée, de toute évidence, par les craintes qui pesaient sur l'existence de la pêche - notamment sédentaire. L'aspect le plus remarquable de cette nouvelle série d'uencouragemens» tient à l'apparition des primes. Après 1763, l'État ne se contenta plus de réduire les impôts qu'il levait sur le commerce du poisson, il versa de l'argent aux armateurs; c'était 
ceux-ci qui allaient maintenant lever un impôt sur l'État. L'initiateur du système des primes fut l'avocat-armateur granvillais Louis Bretel, resté à la Cour depuis sa participation aux négociations franco-anglaises sur Terre-Neuve en 1764. Il présenta dès 1764 au contrôleur général L'Averdy une série de projets d'arrêts: suppression totale de tous les droits perçus sur la morue en France ainsi que dans les colonies françaises, hausse des droits sur la morue étrangère, prime à l'exportation de morue française vers les colonies avec prohibition de la morue étrangère, droits d'exportation symboliques vers l'étranger en attendant la création d'une prime ${ }^{35}$. Bretel estimait que la clé du redressement de la pêche était dans l'extension du marché de la morue sèche aux régions où elle ne pénétrait pas encore: Paris et les provinces de l'est ${ }^{36}$. Il envisageait même de pousser la progression jusqu'en Suisse et en Allemagne: «nous enlèverions aux Hollandois la valleur et les avantages d'une exportation considérable» ${ }^{37}$. L'Averdy se montra favorable à ces suggestions. Malheureusement, «si tout fut promis, rien ne fut exécuté». Le contrôleur général, indisposé par l'opposition de Praslin et de Choiseul à un projet sur l'exportation des eaux-de-vie de cidre, refusa par représailles de signer l'arrêt exemptant la morue française de tous droits d'entrée et de circulation ${ }^{38}$.

Après deux années de vains efforts, Bretel obtint, en mars 1767, l'exemption tant attendue des droits sur la morue sèche à l'entrée de Paris, complétée par une prime de $1 \mathrm{l}$. par quintal introduit ${ }^{39}$. La même année, il gagna sur deux autres «fronts»: il obtint l'attribution de primes pour l'envoi de navires dans les havres nouvellement exploités du French Shore ${ }^{40}$; et il réussit à faire donner aux armateurs une prime d'1 $1.5 \mathrm{~s}$. par quintal de morue envoyé aux Iles ${ }^{41}$. Le problème des exportations de morue aux Iles est une question complexe sur laquelle nous ne pouvons nous étendre $\mathrm{ici}^{42}$. Rappelons simplement que l'approvisionnement des Iles françaises en morue sèche (nourriture des esclaves) fut l'objet d'une compétition entre les négociants de Nouvelle-Angleterre et ceux de France. Le commerce français se trouvait en position de nette infériorité

34 AD Loire-Atlantique, C744, 37, arrêt du Conseil du 8 mai 1742.

35 Archives nationales colonies, C11F 5, 47 et 48, projets d'arrêts par Bretel.

36 AN Colonies, C11F 5, 37, mémoire Bretel 9 mai 1764.

37 AN Colonies, C11F 5, 42, Bretel à Choiseul, 17 novembre 1764.

38 AN colonies, C11F 3, 28, note Bretel, s.d. (entre 1778 et 1783).

39 Ibid.

40 Ces primes se montaient à 1000 livres pour les navires de plus de 60 hommes, 750 1. pour les bâtiments de 41 à 60 hommes et 500 1. pour les navires de moins de 41 hommes. Voir A. Bellet, La Grande Pêche de la morue à Terre-Neuve (Paris, 1902), 59.

41 AD Loire-Atlantique, C744, 38, arrêt du Conseil du 31 juillet 1767.

42 Sur cette question, voir Tarrade, Le Commerce colonial de la France à la fin de l'Ancien Régime: l'évolution du régime de l'Exclusif de 1763 à 1789 (Paris, 1972), 1: chap. VIII. 
du fait que ses terre-neuviers ne pouvaient effectuer le cycle complet France - pêche à Terre-Neuve - Iles - France dans un délai assez court pour réarmer à temps pour la saison suivante. La plupart des envois de morue vers les Iles devaient donc se faire en droiture à partir du Havre, de Nantes ou de Bordeaux. La morue sèche venue de France coûtait ainsi deux ou trois fois plus cher que celle livrée par les Anglo-américains. Fallait-il maintenir le système de l'exclusif? L'administration royale reprochait au commerce de ne pas faire d'envois suffisants vers les Iles; c'est pour cela, disaitelle, qu'on était obligé d'y tolérer les importations de morue étrangère. Les négociants répondaient que c'était justement parce qu'on tolérait ces importations de morue étrangère qu'ils se gardaient d'y envoyer leurs cargaisons, que personne n'achèterait. L'attribution d'une prime et l'interdiction totale d'importer de la morue étrangère aux Iles visait à rompre ce dialogue de sourds. On accorda également aux armateurs le droit d'entrepôt pour leurs sirops et tafias reçus en paiement des morues ${ }^{43}$. En 1768, afin d'inciter le commerce à suivre la bonne voie, le gouvernement finança (en secret) l'armement d'un bâtiment chargé de morues pour la Martinique. L'échec de ces mesures fut total. En 1772, Bretel demandait, sans succès, le triplement de la prime ${ }^{44}$.

À partir de 1773, toutefois, «les espérances du commerce de la pesche commencèrent à renaître ${ }^{45}$. Le droit de consommation sur les morues sèches fut réduit de moitié et la réduction des droits d'entrée octroyée en 1742 fut étendue à tous les ports de Norman$\mathrm{die}^{46}$. Deux ans plus tard, Bretel obtenait enfin l'exemption de tous droits royaux sur la morue sèche ${ }^{47}$. "Vous ne sauriez croire, Messieurs, écrit-il, combien le terrain m'a esté disputé par la Ferme Générale. ${ }^{48}$ Celle-ci parvint à empêcher l'extension de la franchise aux morues vertes sous prétexte que l'abolition des contrôles favoriserait la contrebande du sel ${ }^{49}$.

$\mathrm{Au}$ lendemain de la guerre d'Indépendance américaine, une nouvelle et dernière étape s'entama au cours de laquelle l'attribution des primes supplanta définitivement celle des exemptions. Le gouvernement accorda aux armateurs en 1784 - toujours à la

43 AN Colonies, C11F 5, 3, fo 29, arrêt du Conseil du 14 mars 1767.

44 AN Colonies, C11F 5, 70, note Bretel 1772.

45 AN Colonies, C11F 3, 31, note Bretel, s.d. (1778-1783). bre 1773 .

46 AD Loire-Atlantique, C744, 37, arrêts du Conseil des 24 mars et 19 septem-

47 AD Loire-Atlantique, C744, 35, arrêt du Conseil du 30 janvier 1775.

48 AN Marine, C5, 46, Bretel aux armateurs malouins et granvillais, 5 novembre 1772.

49 AD Loire-Atlantique, C744, 111, Chambre de Commerce de Nantes à Sartine, 3 août 1775. Les Nantais sont furieux: «les soupçons mal fondés et la mauvaise humeur des fermiers doivent être sacrifiés à l'intérêt immédiat de la pêche nationale.» 
demande de Bretel - une prime de 100 1. par homme d'équipage envoyé sur la côte occidentale de Terre-Neuve. Cette gratification, ramenée à $75 \mathrm{l}$. l'année suivante et en principe temporaire, fut donnée jusqu'en $1792^{50}$. En 1785, on admit la morue anglaise et américaine aux Iles moyennant un droit de $5 \mathrm{l}$. par quintal; en échange, la prime reçue par la morue française fut élevée au taux énorme de 10 1. par quintal. Suivant la suggestion déjà ancienne de Bretel, on utilisait les droits versés par les Anglo-américains pour payer les primes aux armateurs français ${ }^{51}$. Les envois de morue française étant restés médiocres, il fallut, en 1787, hausser la prime à $12 \mathrm{l}$. et les droits sur le poisson étranger à $8 \mathrm{l}$. par quintal. En 1785, Bretel obtint également l'attribution d'une prime de 5 1. par quintal exporté en Europe ${ }^{52}$. Deux ans plus tard, on étendit le bénéfice de cette mesure aux morues exportées vers les ports de la Méditerranée orientale ${ }^{53}$.

En l'espace d'un siècle, s'opéra ainsi une sorte de renversement dans la manière dont l'État concevait ses rapports avec le commerce de la morue. On était parti d'un système dans lequel l'attention portée à ce commerce tenait essentiellement à sa place dans l'édifice fiscal. Le commerce de la morue soutenait par l'impôt l'État prédateur. Puis, progressivement, sous la pression convergente de multiples facteurs, cette approche fiscaliste se modifia, en trois étapes successives: exemptions de droits avant la guerre de Sept ans; exemptions et primes entre les guerres de Sept ans et d'Amérique; primes seulement après 1783. L'État rançonneur et passablement indifférent du siècle précédent laissait place à un État manipulateur, qui contrôlait, dirigeait, orientait, ou tentait de le faire. En décidant en 1785 de ne plus calculer le montant des prises de la pêche française aux ports de déchargement des cargaisons mais aux ports d'armement des navires, le gouvernement entérinait en quelque sorte ce renversement: l'État ne s'intéressait plus à ce que chaque port avait reçu - approche fiscaliste - mais à ce que chaque port produisait - approche dirigiste ${ }^{54}$. Le projecteur statistique déplaçait son faisceau sur un type d'informations dont la possession allait accroître les possibilités de contrôle et d'action du gouvernement sur la pêche terre-neuvière.

En laissant aux armateurs terre-neuviers la charge de former des hommes que la rivalité franco-anglaise rendait de plus en plus précieux, le gouvernement royal donnait au commerce des moyens

\footnotetext{
AN Marine, C5, 52, De Castries à Chambre de Commerce, 25 décembre 1785. AD Loire-Atlantique, C744, arrêt du Conseil du 18 septembre 1785. Ibid.

AD Loire-Atlantique, C744, 31, arrêt du Conseil du 11 février 1787.

AN Marine, C5, 52, De Castries aux commissaires des classes, 31 mars 1785.
} 
de pression que celui-ci ne se fit pas faute d'utiliser pour obtenir les avantages qu'il recherchait. Mais en retour, ces avantages devinrent eux-mêmes des moyens de pression et de manipulation sur le commerce terre-neuvier. Sous la menace d'un abandon toujours possible de la pêche terre-neuvière, les armateurs forcèrent l'État à les aider. Comme l'État, de son côté, pouvait à tout moment cesser ce soutien, les armateurs durent accepter, non sans rechigner, le dirigisme croissant qui en fut le prix. 